\title{
Analysis for Difficulty during Freeze-Drying Feizixiao Lychees
}

\author{
L. L. Huang, F. Qiao, G. Peng, and C. F. Fang \\ School of Applied Chemistry and Biological Technology, Shenzhen Polytechnic, Shenzhen, Guangdong 518055, China \\ Correspondence should be addressed to F. Qiao; qiaofang@szpt.edu.cn
}

Received 23 June 2017; Revised 29 August 2017; Accepted 13 September 2017; Published 24 December 2017

Academic Editor: Hui-Min D. Wang

Copyright (c) 2017 L. L. Huang et al. This is an open access article distributed under the Creative Commons Attribution License, which permits unrestricted use, distribution, and reproduction in any medium, provided the original work is properly cited.

\begin{abstract}
Compared to other cultivar lychees, volume density of Feizixiao lychee was higher due to serious shrinkage during freeze-drying (FD). Guiwei lychee and Nuomici lychee were used for comparison in order to illuminate the reason of the aforementioned phenomenon. Lower prefreezing temperature could not improve the volume density of Feizixiao lychee. Microstructure results show that pulp cell of Feizixiao lychee (tail) was smaller and more compact than Guiwei and Nuomici lychee pulp cell. In addition, there is a membrane around the surface of Feizixiao lychee pulp. And the microstructure of Feizixiao lychee tip pulp cell is different from tail pulp cell. Membrane and tip pulp cell are both smaller and more compact than tail pulp cell. These structure differences hinder the moisture removing of Feizixiao lychee during FD. Removing the membrane and tip pulp could not improve the volume density of Feizixiao lychee. Ultrasound treatment for $30 \mathrm{~min}$ could significantly enhance the volume density of Feizixiao lychee.
\end{abstract}

\section{Introduction}

Lychee (Litchi chinensis Sonn.), one of the major fruits in South China, is liked for its characteristic sweet acidic taste, excellent aroma, high nutrient value, and the attractive deep bright red color of the pericarp $[1,2]$. Shenzhen Nanshan lychees, including Feizixiao, Guiwei, and Nuomici, were recognized as product of geographical indication in 2006. Lychees are a seasonal fruit. At present, lychees are commonly precooled first and then put together with ice bag during transport in order to keep the original quality of lychees. In addition, fresh-keeping agent coating and soaking are also common methods for lychee preservation [3-5]. So far, the storage life of fresh lychees barely exceeds one month even using the best preservation method according to previous studies. Therefore, the processing and preservation of lychees are very imperative.

Now, dried lychees account for $80 \%$ of all lychee processing products [6], where air dried lychees are the major. Air dried lychee pulp is brown with serious shrinkage, which limit the application of air dried lychees. Moreover, most air dried lychees are whole lychees and they need to be peeled and have their stones removed before using. Lychee pulp after air drying is tightly bound to kernel and the pulp is sticky due to high sugar content and 25-30\% moisture content, which make the kernel removing difficult. In recent years, microwave was applied to dry lychees, including microwave air drying and microwave vacuum drying [1]. Microwave using could decrease the energy consumption and increase the quality of product [7]. However, these drying methods with microwave were not applied widely due to nonuniformity of microwave and other equipment problems. It is well known that freeze-drying (FD) can maintain the maximum original quality of materials $[8,9]$. It is found that Feizixiao lychee showed serious shrinkage during FD but Guiwei and Nuomici lychee could be freeze dried well. This was an interesting phenomenon and deserved further research.

A successful FD process retains the volume of the material. There are many reasons which affect the volume of samples during FD, like prefreezing temperature, heating plate, sugar content of materials, and so forth. Drying temperatures below the temperature of glass transition during heating allow removal of ice within the solid. But temperatures above ice melting temperature can result in collapse [10]. The structure of materials containing sugars may collapse during dehydration if the temperature of ice within the material is higher than the collapse temperature [11]. Lychee pulp contains high sugars including sucrose, fructose, and glucose $[12,13]$ and the sugar content is different with lychee 
cultivar. There is a close relationship among these influence factors. Sugar content decides the prefreezing temperature and prefreezing and heating plate temperature affect the drying temperature of materials during FD. Differential scanning calorimetry (DSC) can be used to measure eutectic point temperature $\left(T_{g}\right)$ of materials [14]. Prefreezing temperature should be confirmed according to $T_{g}$.

Microstructure of food could reflect the macroscopic quality of food [15]. Macroscopic shrinkage of materials essentially resulted from breaking and collapse of cell structure [16-18]. Huang et al. reported that lychee pericarp consisted of three parts: outer layer with cuticle, interlayer, and inner layer. Inner layer cells are small and intact, which hinder the moisture removing during drying. It shows that cell structure of materials could significantly affect the drying process. Although peel lychee was used during FD, there may be significant differences about cell structure of lychee pulp among different cultivars. In fact, there is a film around the surface of Feizixiao lychee pulp but not Guiwei and Nuomici lychee. Moreover, there is residue in mouth after eating Feizixiao lychee pulp, especially tip pulp. But Guiwei and Nuomici lychees have no residue. These macroscopic differences may result from microstructure differences of lychee pulp with different cultivar. Therefore, it is necessary to analyze and compare the microstructure of three cultivar lychees' pulp.

In this research, authors not only clarify the difficulty of Feizixiao during FD, but also find out the effective method to resolve the problem. Ultrasound is usually used in food process to improve the quality of food, such as ultrasoundassisted freezing and ultrasound pretreatment. Ultrasound can enhance nucleation rate and crystal growth rate and improve the physicochemical properties of freezing red radish [19]. Ultrasound pretreatment can enhance the glass transition temperature of pear during FD [20]. The more the ultrasonic power application, the higher the glass transition temperature. It indicated that ultrasound pretreatment prior to freeze-drying can improve the efficiency of FD and the stability during storage of FD pear. Ultrasound pretreatment did reduce drying time by $13-17 \%$, increased the lightness, decreased the brown pulp, and improved the rehydration properties of dried apple [21].

The objectives of this work were to analyze the difficulty of Feizixiao during FD compared with Guiwei and Nuomici lychee, to improve the FD Feizixiao lychee quality by ultrasound pretreatment.

\section{Materials and Methods}

2.1. Samples. Fresh lychee fruits (Litchi chinensis Sonn.) cv. Feizixiao, Nuomici, and Guiwei at commercially mature stage were picked from Xili orchard, Shenzhen, China (east longitude: $113^{\circ} 56^{\prime} 01.16^{\prime \prime}$, north latitude: $\left.22^{\circ} 36^{\prime} 15.26^{\prime \prime}\right)$. Mature lychee fruits that were free from visible blemish or disease were selected. Every lychee fruit weight was about $25 \mathrm{~g}$. The average sizes (horizontal $\times$ vertical) of Feizixiao, Nuomici, and Guiwei lychees were $37.48 \mathrm{~mm} \times 38.20 \mathrm{~mm}, 38.00 \mathrm{~mm}$ $\times 35.77 \mathrm{~mm}$, and $36.91 \mathrm{~mm} \times 35.64 \mathrm{~mm}$. The picture of three fresh lychees was shown in Supplementary Materials. Lychees
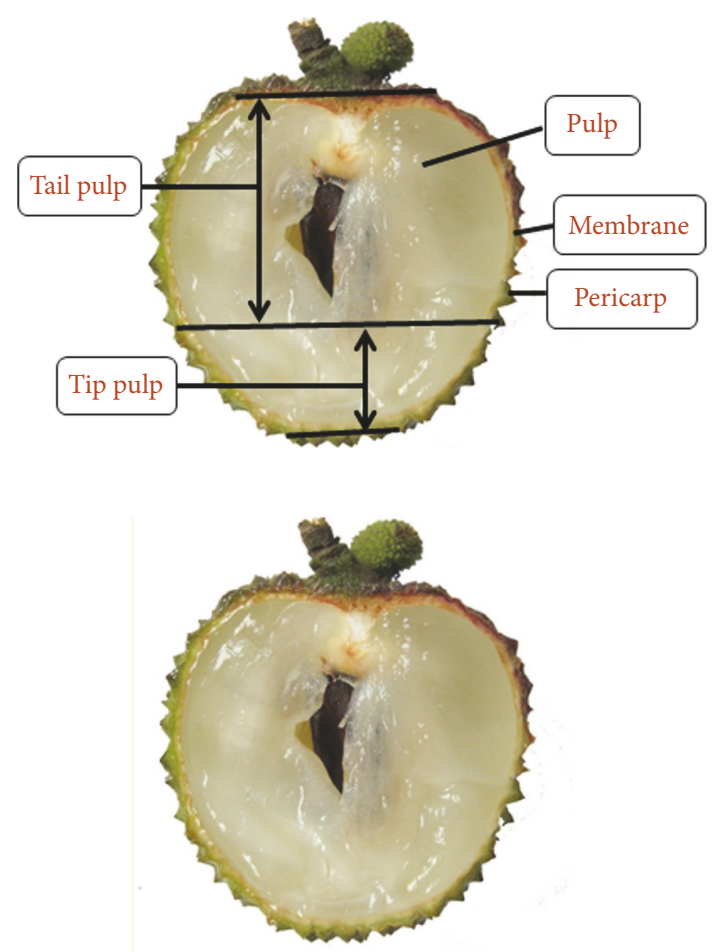

FIgURE 1: The schematic diagram of different part in Feizixiao lychee.

were washed, peeled, and pitted and then every lychee pulp was divided into two parts before prefreezing.

2.2. Equipment and Drying Experiment. The lab-scale vacuum freeze dryer was made by Alpha 1-4 LSC freezer (Marin Christ Company, Osterode, Germany). The minimum absolute pressure of freeze dryer is $1 \mathrm{~Pa}$ and the lowest temperature of cold trap is $-55^{\circ} \mathrm{C}$. The temperature of heating plate during the second stage was $60^{\circ} \mathrm{C}$. Lychees were dried by FD until the final moisture content was less than $10 \%$ (wet basis).

Two pieces of freezing equipment were used for prefreezing in this study. One was freezer with the $-30^{\circ} \mathrm{C}$ (Kelen business equipment Co. Ltd., Shenzhen, China) and another was $-80^{\circ} \mathrm{C}$ ultra-low temperature freezer (EXF32086V, Thermo Co. Ltd., Waltham, MA, America).

2.3. Ultrasound Treatment. An ultrasound-microwave synergistic extraction apparatus (CW-2000, Shanghai Xintuo Analytical Instrument Co. Ltd., Shanghai, China) was applied for lychee pulp. Only ultrasound with $40 \mathrm{kHz}$ and $50 \mathrm{~W}$ was used during experiment. The ratio of lychee pulp to water was $1: 4$ and the experiment temperature was $30^{\circ} \mathrm{C}$. Different treatment time $(10 \mathrm{~min}, 20 \mathrm{~min}$, and $30 \mathrm{~min})$ was studied. Lychees that were washed, peeled, and pitted with every lychee pulp divided into two parts were control group. For some special samples, membrane was moved by hand after being peeled and tip pulp was cut by knife after being pitted. The schematic diagram of different part in Feizixiao lychee was shown in Figure 1. The experiment was replicated three times. 
TABLE 1: Results of three indexes for three cultivar lychees' pulp.

\begin{tabular}{lccc}
\hline Cultivar & Volume density $\left(\mathrm{g} / \mathrm{cm}^{3}\right)$ & Eutectic point $\left({ }^{\circ} \mathrm{C}\right)$ & Pectin content $(\mathrm{g} / \mathrm{kg})$ \\
\hline Feizixiao & $0.754 \pm 0.020^{\mathrm{a}}$ & $-26.80 \pm 0.28^{\mathrm{c}}$ & $2.115 \pm 0.083^{\mathrm{a}}$ \\
Guiwei & $0.495 \pm 0.032^{\mathrm{b}}$ & $-16.32 \pm 0.61^{\mathrm{a}}$ & $1.486 \pm 0.061^{\mathrm{c}}$ \\
Nuomici & $0.462 \pm 0.024^{\mathrm{b}}$ & $-20.30 \pm 0.31^{\mathrm{b}}$ & $1.836 \pm 0.091^{\mathrm{b}}$ \\
\hline
\end{tabular}

Values in the same column not sharing the same superscript are significantly different $(p<0.05)$.

2.4. Microstructure. Structures of lychee pulp were studied using light microscopy (LM). The paraffin method was used [22]. Small cubes (pulp: about $4 \mathrm{~mm}^{3}$, pericarp: about $2 \mathrm{~mm}^{2}$ ) were removed from the internal zone of the samples for microscopic examination. The sample cubes were fixed in formol-aceto-alcohol (FAA, formaldehyde 5\%, glacial acetic acid $5 \%$, and $70 \%$ ethanol $90 \%$ ) fixative solution for 24 hours. The ratio of fixative solution to samples was about $30: 1$. Dehydration was performed with $70 \%, 85 \%$, and $95 \%$ ethanol concentrations for $2 \mathrm{~h}$, respectively, and $100 \%$ ethanol concentration for $50 \mathrm{~min}$. Then samples were cleared in mixed solution (ethanol:xylene $=1: 1$ ) and pure xylene solution for $2 \mathrm{~h}$, respectively. Samples with xylene solution were put in China cups and melted paraffin was added in the cups $(1: 1)$. The cups were put in incubator at $40^{\circ} \mathrm{C}$ for $24 \mathrm{~h}$. Next the cups were put in incubator at $60^{\circ} \mathrm{C}$ and paraffin was dumped after melting. Paraffin was added again in cups for one-hour interval and then dumped for three times. Last samples were embedded in paraffin (melting points $55^{\circ} \mathrm{C}$ to $\left.57^{\circ} \mathrm{C}\right)$.

Sectioning was done with a rotary microtome (RM 2126, Shanghai Leica Instruments Ltd., China) at $10 \mu \mathrm{m}$ thickness. Sections were stuck on microslides with gelatin adhesive. After removing the paraffin from samples with xylene for $10 \mathrm{~min}$, the sections were rehydrated with a series of decreasing ethanol steps $(100 \%, 95 \%, 85 \%$, and $70 \%)$ for $10 \mathrm{~min}$, respectively, and then the Heidenhain's iron-alum hematoxylin method was employed for staining. Finally, the samples were examined under a light microscope (Eclipse 50i, Shanghai Nikon Instruments Inc., China) equipped with a digital camera (DS-Fil, Shanghai Nikon Instruments Inc., China). The histological procedures were performed in duplicate.

2.5. Pectin. Pectin was measured by carbazole spectrophotometric determination method (NY/T82.11-1988). Results of pectin were expressed as galacturonic acid (GlaA) g/100 g of initial fresh sample.

2.6. Eutectic Point. The eutectic point temperature of lychee was conducted with a differential scanning calorimeter (DSC, Q2000, TA Instruments, New Castle, DE) by Syamaladevi et al. [23] Lychee pulp was broken and then centrifuged with $8000 \mathrm{rpm} / \mathrm{min}$ at $4^{\circ} \mathrm{C}$. Lychee pulp juice was used to test eutectic point temperature. Following equilibration, $15-20 \mathrm{mg}$ lychee juice was sealed in aluminum pans $(2 \mathrm{~mm} \times$ $5 \mathrm{~mm} \times 7 \mathrm{~mm}, 10-12 \mathrm{mg}$ ) and cooled from room temperature to $-90^{\circ} \mathrm{C}$ at $5^{\circ} \mathrm{C} / \mathrm{min}$ and equilibrated for $10 \mathrm{~min}$. Lychee juice was scanned from $-90^{\circ} \mathrm{C}$ to $50^{\circ} \mathrm{C}$ at a rate of $5^{\circ} \mathrm{C} / \mathrm{min}$ $\left(T_{m}\right)$. Annealing procedure was subjected to annealing at a temperature $T_{m}$ for $30 \mathrm{~min}$ during decreasing temperature process. Then samples were cooled from $T_{m}$ to $-90^{\circ} \mathrm{C}$ at $5^{\circ} \mathrm{C} / \mathrm{min}$ and equilibrated for $10 \mathrm{~min}$. And samples were scanned from $-90^{\circ} \mathrm{C}$ to $50^{\circ} \mathrm{C}$ at a rate of $5^{\circ} \mathrm{C} / \mathrm{min}$.

2.7. Bulk Density. Spiked millet substitution method was used to detect the volume of dried mixed chips. The granularity of spiked millet is between 0.9 and $1.1 \mathrm{~mm}$ [24]. The experiment was replicated three times. The bulk density $\rho$ of the dried material is defined as

$$
\rho=\frac{m}{V},
$$

where $m$ is the mass of dry chips and $V$ is total volume of dried mixed chips.

2.8. Statistical Analysis. The experimental data was analyzed using the statistical software SPSS 18 (SPSS Inc. Chicago, IL, USA) and analyses of variance were conducted by ANOVA procedure. All the measurements were carried out in triplicate. Mean values were considered significantly different when $p \leq 0.05$.

\section{Results and Discussion}

3.1. Effect of Prefreezing Temperature on FD Feizixiao Lychee. Volume density values of three FD lychees were shown in Table 1. It can be seen that the volume density of Feizixiao was high due to serious shrinkage. The picture of FD Feizixiao lychees was shown in Supplementary Materials. Effect of prefreezing temperature on FD process is very important. The temperature of materials should be under eutectic point temperature during prefreezing process, which will result in freeze completely. From Table 1, it can be observed that there were significant differences about eutectic point temperature among three cultivar lychees (Figure 2). And eutectic point temperature of Feizixiao lychee was lower than that of Guiwei and Nuomici lychees. Sugar content, sugar composition, and each ratio of three lychee juice could explain this phenomenon.

The results of sugar content and sugar composition of three lychees were shown in Figure 3. It can be seen that the total sugar content of Feizixiao was the highest. The higher the sugar content, the lower the eutectic point temperature of materials [25]. Moreover, lychee pulp contains three sugars: glucose, fructose, and sucrose. And each sugar ratio was different among three lychees. Sucrose content was lower and glucose and fructose content was higher in Feizixiao lychee juice. It is well known that eutectic point of Invertose is lower than sucrose [26]. Therefore, higher total sugar content and 


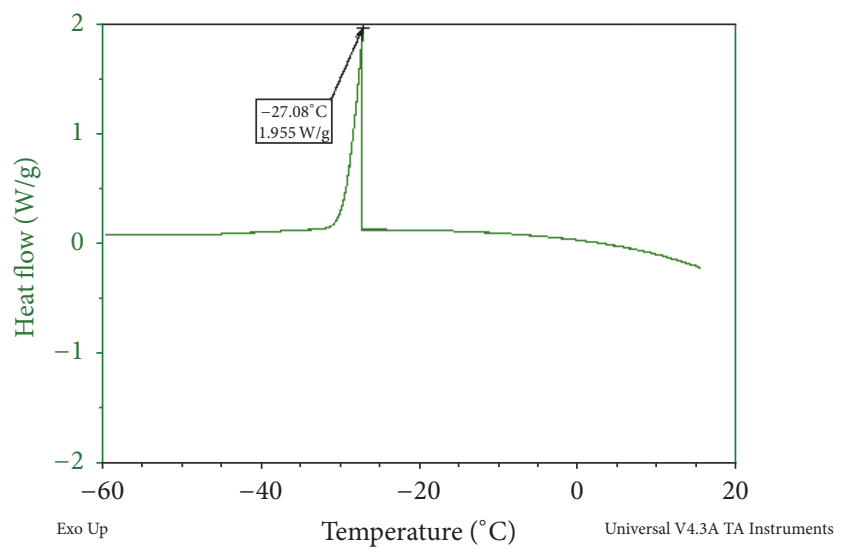

(a)

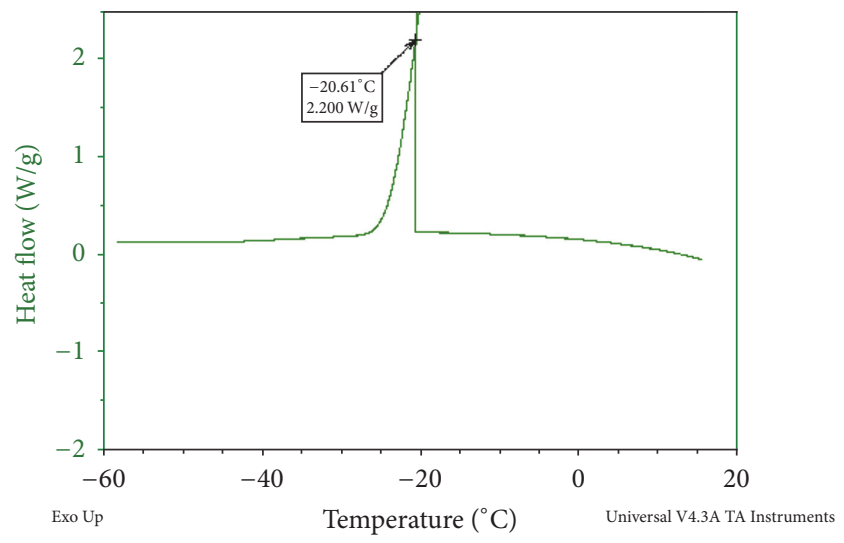

(b)

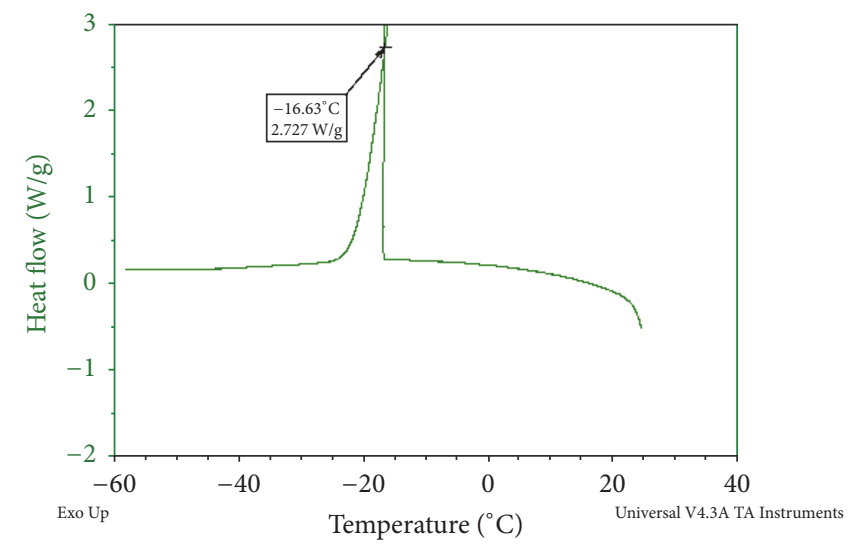

(c)

FIGURE 2: DSC curve for pulp clear juice of three lychees. (a) Feizixiao lychee. (b) Nuomici lychee. (c) Guiwei lychee.

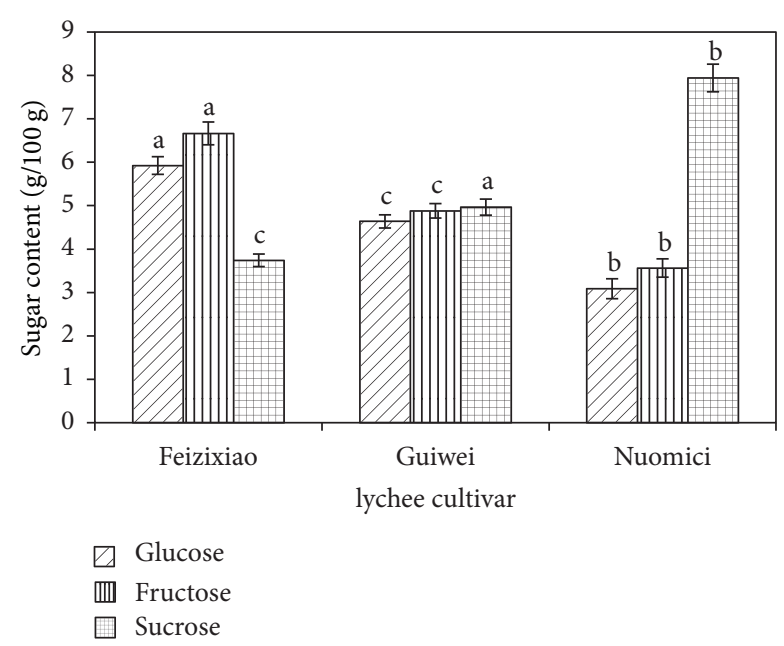

FIGURE 3: Sugar compositions in three cultivar lychees. ((a), (b), (c)) Values in the same column not sharing the same superscript are significantly different $(p<0.05)$.

high Invertose content were the main reason for low eutectic point temperature of Feizixiao juice.

In general, freezing temperature is $5^{\circ} \mathrm{C}-10^{\circ} \mathrm{C}$ below eutectic point temperature at least $[27,28]$. However, the prefreezing temperature of three cultivar lychees was $-30^{\circ} \mathrm{C}$ before this study. Guiwei and Nuomici lychees can freeze completely at this temperature. But for Feizixiao lychee, this temperature did not meet the freeze complete demand. So, the prefreezing temperature of Feizixiao should be decreased. The prefreezing temperature was $-50^{\circ} \mathrm{C}$ in order to analyze other factors during follow-up tests.

3.2. Effect of Microstructure on FD Feizixiao Lychee. The volume density of Feizixiao lychee was also high with serious shrinkage even after $-50^{\circ} \mathrm{C}$ prefreezing temperature. In fact, the microstructure plays an important role in determining the quality of materials $[17,18]$. So, microstructure of Feizixiao lychee was tested for analysis. The micrographs of three lychee pulps were shown in Figure 4. It can be observed that the cell size of Guiwei and Nuomici lychee pulp was very close. But the cell size of Feizixiao lychee pulp was much smaller than the other two lychees. In general, lychee pulp was composed of long tubular structure including many single polygon cells. The moisture of lychee pulp is removed out by cell pore. The smaller the cell size, the higher the removing resistance of moisture [22]. This should be another reason for difficulty during freeze-drying Feizixiao lychees.

There is a translucent coating around the surface of Feizixiao lychee pulp but Guiwei and Nuomici lychees have 


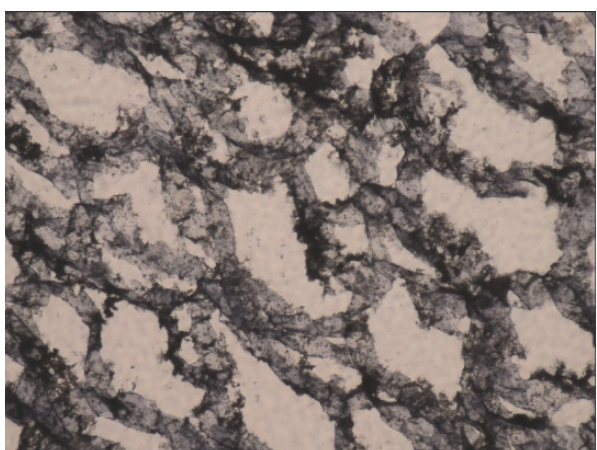

Nuomici pulp 200x

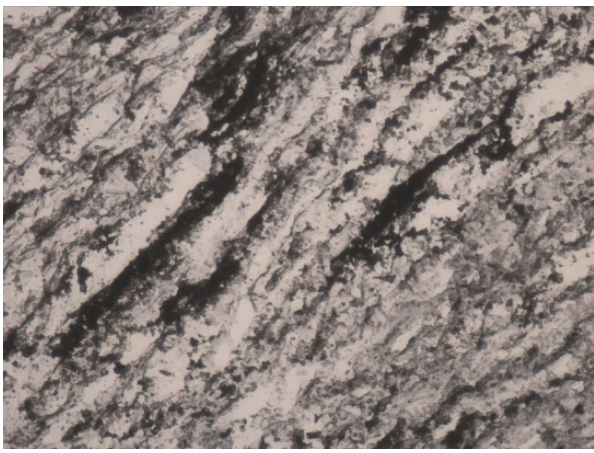

Feizixiao pulp 200x

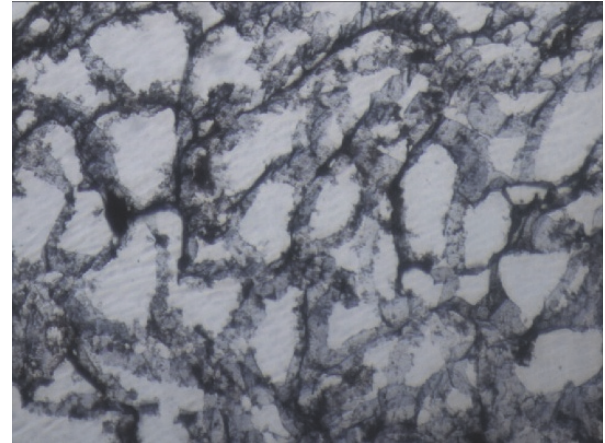

Guiwei pulp 200x

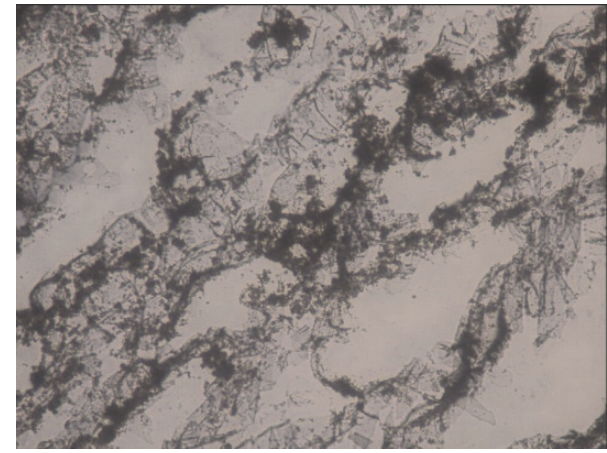

Feizixiao pulp 400x

FIGURE 4: The light micrographs for lychee pulp of three cultivars.

not. Moreover, the color and taste of tip pulp are different from that of tail pulp. Tip pulp has slight red color and residue feeling after taste, which means that there is significant difference between tip and tail pulp of Feizixiao lychee. The micrographs of coating and tip pulp in Feizixiao lychee were shown in Figure 5. It can be seen that the cell structure of coating was small and intact, which increases the removing resistance of moisture in pulp. The tip cell was smaller and more compact than tail pulp cell. In addition, random arrangement with more folding not long tubular structure arrangement appears in tip pulp, which results in more difficult moisture removing from tip pulp. In a word, microstructure of Feizixiao pulp impedes the moisture removing during FD.

It is reported that the cell wall of potato was more resistant and stable after soaking with $\mathrm{CaCl}_{2}$ [29]. It can be explained that pectin was combined with $\mathrm{Ca}^{2+}$ and the thickness of cell wall was increased accordingly. In this study, Feizixiao lychee pulp was soaked with $0.5 \%$ and $1 \% \mathrm{CaCl}_{2}$ solution. However, shrinkage of Feizixiao lychee was not improved like expected. The results of pectin content in three lychees were shown in Table 1. It can be seen that the pectin content in Feizixiao lychee was the highest. Eutectic point temperature of lychee juice will decrease with soluble pectin content increasing [25]. This was another reason for low eutectic point temperature of Feizixiao lychee juice except for sugar.

3.3. Effect of Ultrasound on FD Feizixiao Lychee. As known above, microstructure of Feizixiao lychee pulp affects the volume density of FD pulp. Ultrasound treatment can decrease the drying time and increase the quality of dried samples $[21,30,31]$. So, ultrasound was applied to treat Feizixiao lychee pulp in order to improve the appearance of FD pulp. The volume density results of Feizixiao lychees are treated by ultrasound in Figure 6. And the volume density results of Guiwei and Nuomici lychees are treated by ultrasound in Figure 7. Figure 6 shows that ultrasound could significant decrease the volume density of Feizixiao pulp. And the appearance of samples without membrane and tip pulp was much better than control group. Photos of Feizixiao lychee sample treated by ultrasound were shown in Supplementary Materials. It may be because the suitable ultrasound treatment could improve the permeability of cell wall, which results in more easy moisture removing. There was no significant difference about volume density between $20 \mathrm{~min}$ and $30 \mathrm{~min}$ treatment. This can be explained by the fact that there was similar permeability increase induced by two ultrasound treatments with different time. If the treatment time was prolonged continuously, the permeability of pulp cell wall will be improved further or decreased instead (Figure 7). However, even if the permeability of cell wall is increasing with treatment time, too much soluble solid loss resulting therefrom will appear. Therefore, the optimal ultrasound treatment time was $20 \mathrm{~min}$.

The appearance of Guiwei and Nuomici lychee was improved further by ultrasound treatment. The optimal treatment time was also $20 \mathrm{~min}$. It is worth noting that the volume density of Guiwei lychee pulp increased after 30 min ultrasound treatment. It may be because ultrasound treatment that is too long aggravates the breakage of cell wall. 


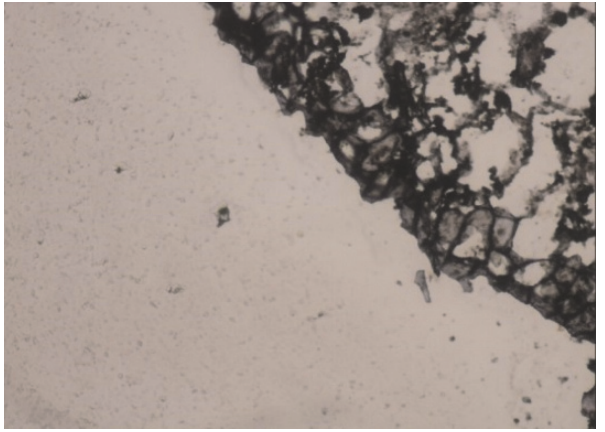

Feizixiao pulp membrane 200x

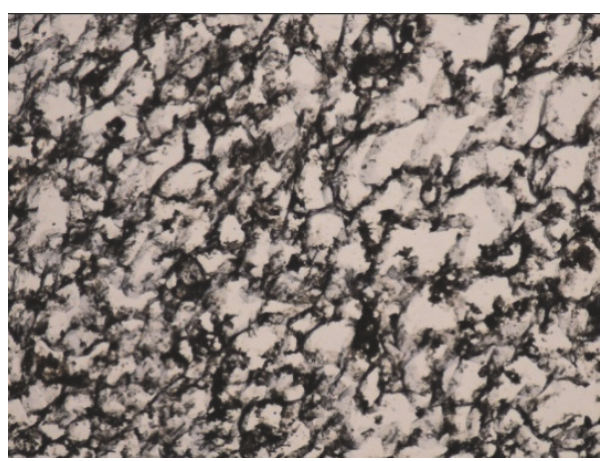

Feizixiao pulp tip 200x

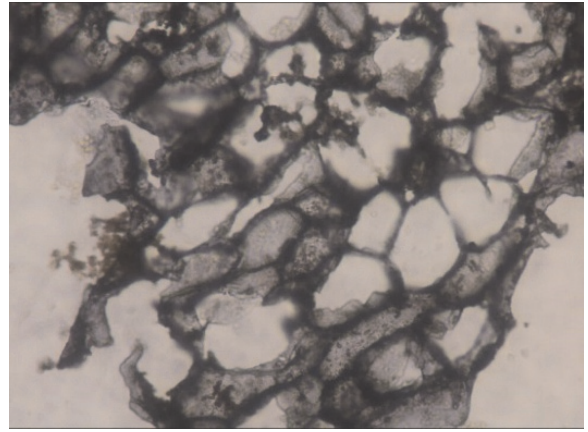

Feizixiao pulp membrane 400x

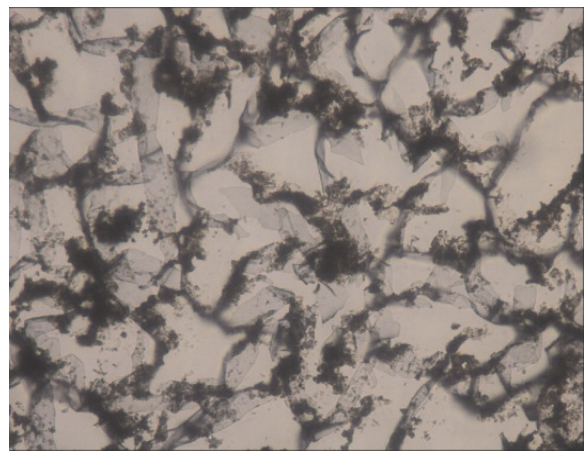

Feizixiao pulp tip 400x

FIGURE 5: The light micrographs for Feizixiao lychee pulp membrane and tip.

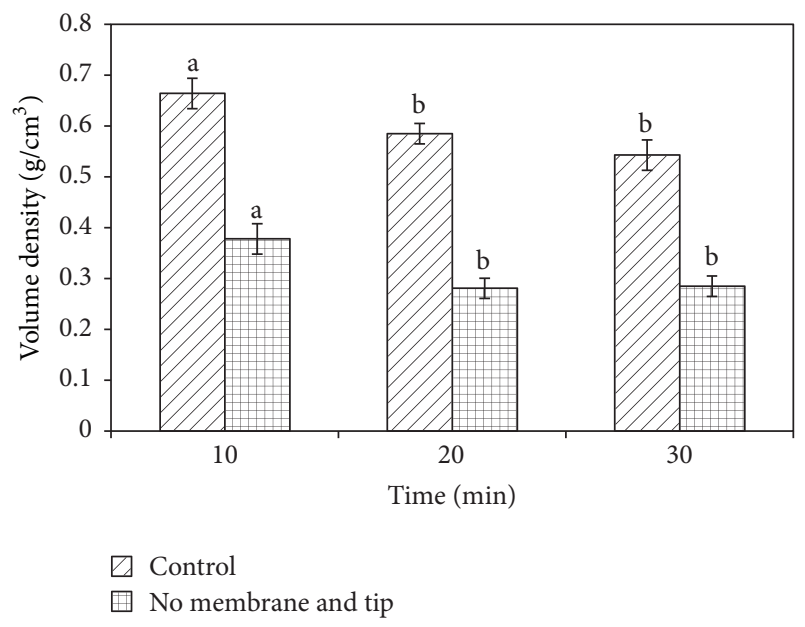

FIgURE 6: Effect of ultrasound on volume density of FD Feizixiao lychee. ((a), (b), (c)) Values in the same column not sharing the same superscript are significantly different $(p<0.05)$.

Then fragment appearing and folding impede the moisture removing of lychee pulp. Photos of Nuomici and Guiwei samples treated by ultrasound for 30 min were also shown in Supplementary Materials.

\section{Conclusions}

Although Feizixiao lychee is one of the major lychee cultivars in Nanshan, Shenzhen, its drying characteristic is

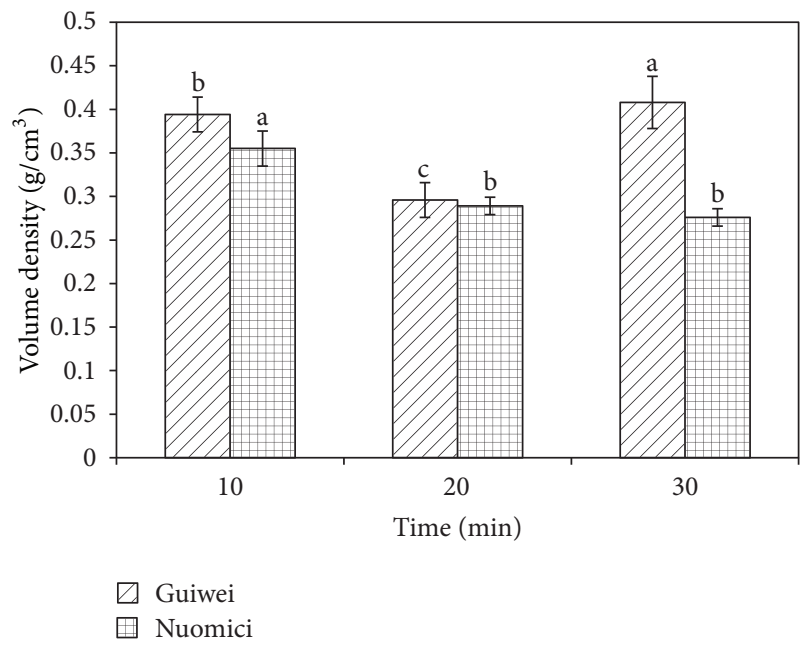

FIGURE 7: Effect of ultrasound on volume density of FD Guiwei and Nuomici lychee. ((a), (b), (c)) Values in the same column not sharing the same superscript are significantly different $(p<0.05)$.

different from the other two major cultivars: Nuomici and Guiwei. It needs lower prefreezing temperature for Feizixiao lychees during freeze-drying due to higher sugar content and more Invertose content. Prefreezing temperature of Feizixiao lychee pulp should be below $-32^{\circ} \mathrm{C}$ at least. For lychee pulp microstructure, the cell of Feizixiao lychee pulp is smaller and tighter than Guiwei and Nuomici lychees. In addition, the structure of Feizixiao lychee pulp is more complex than 
Guiwei and Nuomici lychee pulp. A translucent membrane is around the surface of Feizixiao pulp. And the microstructure of tip pulp is different from the tail pulp for Feizixiao lychees. The cell of tip pulp is smaller than that of tail pulp. The membrane and smaller cell of pulp are both factors for difficulty during FD Feizixiao lychees due to hard moisture removing.

Ultrasound treatment can improve the appearance of FD Feizixiao lychee pulp, especially for samples without membrane and tip pulp. Very long treatment time could decrease the appearance of FD Feizixiao lychee pulp. Therefore, a suitable treatment time is very important. Moreover, removing the membrane and tip pulp not only is very trouble work, but also wastes materials. Therefore, new treatment methods will be quested in the future for high quality FD Feizixiao whole pulp with membrane and tip pulp.

\section{Additional Points}

Practical Applications. At present, air dried (AD) lychees are popular as traditional food in South China. The yield of AD lychees is higher than freeze-drying (FD) lychees. AD lychee has deep brown pulp with much shrinkage and poor flavour. However, the application of FD lychees increases constantly due to white color, crisp taste, and little shrinkage. For example, FD lychee powder could be added in cookies or desserts. Feizixiao lychee is one of the major lychee cultivars in China. This research could resolve the shrinkage of Feizixiao lychee during FD, which will improve the processing property and application of Feizixiao lychee.

\section{Conflicts of Interest}

The authors declare that there are no conflicts of interest regarding the publication of this paper.

\section{Acknowledgments}

The authors acknowledge China Modern Agro-Industry Technology Research System (no. CARS-33-20) and National Natural Science Foundation of China (no. 31671907).

\section{Supplementary Materials}

The FD lychee photos of three cultivars were shown. It can be seen that FD Feizixiao lychees have significant shrinkage. Feizixiao lychees with ultrasound treatment for $30 \mathrm{~min}$ have better appearance. Feizixiao lychees with no membrane and tip have much better appearance. The samples with best appearance were Feizixiao lychees with no membrane and tip and ultrasound treatment for $30 \mathrm{~min}$. Shenzhen Nanshan lychees, including Feizixiao, Guiwei, and Nuomici, were recognized as product of geographical indication in 2006. Three cultivar lychees are all main cultivars in Guangdong province. They are different from each other in appearance including color, shape, and surface structure. (Supplementary Materials)

\section{References}

[1] X. Duan, L.-L. Huang, M.-M. Wang, F. Qiao, and C.-F. Fang, "Studies on the Effects of Microwave Power and Temperature Control on the Quality of Whole Lychee (Litchi chinensis Sonn.) Fruit during Microwave Vacuum Drying," Journal of Food Processing and Preservation, vol. 39, no. 4, pp. 423-431, 2015.

[2] N. S. Shah and N. Nath, "Changes in qualities of minimally processed litchis: Effect of antibrowning agents, osmo-vacuum drying and moderate vacuum packaging," LWT-Food Science and Technology, vol. 41, no. 4, pp. 660-668, 2008.

[3] S. Yang, Y. Chen, L. Feng, E. Yang, X. Su, and Y. Jiang, "Effect of methyl jasmonate on pericarp browning of postharvest lychees," Journal of Food Processing and Preservation, vol. 35, no. 4, pp. 417-422, 2011.

[4] S. P. Yang, J. Xie, Y. F. QIan et al., "Preservation of litchi with composite chitosan coating and modified atmosphere packaging," Food Science, vol. 34, no. 8, pp. 279-283, 2013 (Chinese).

[5] Y. Mo, J. Zheng, W. Li, T. Niu, and J. Xie, "Effects of ascorbic acid and glutathione treatments on litchi fruits during post harvest storage," Transactions of the Chinese Society Agricultural Engineering, vol. 26, no. 3, pp. 363-368, 2010 (Chinese).

[6] F. Qiao, L.-L. Huang, and W.-S. Xia, "A study on microwave vacuum dried re-structured lychee (Litchi chinensis Sonn.) mixed with purple sweet potato (Ipomoea batatas) snacks," Food and Bioproducts Processing, vol. 90, no. 4, pp. 653-658, 2012.

[7] D. M. Fan, L. Y. Wang, N. N. Zhang et al., "Full-time response of starch subjected to microwave heating," Scientific Reports, vol. 7, no. 3967, 2017.

[8] F. Pei, Y. Shi, A. M. Mariga et al., "Comparison of Freeze-Drying and Freeze-Drying Combined with Microwave Vacuum Drying Methods on Drying Kinetics and Rehydration Characteristics of Button Mushroom (Agaricus bisporus) Slices," Food and Bioprocess Technology, vol. 7, no. 6, pp. 1629-1639, 2014.

[9] A. Tarafdar, N. C. Shahi, A. Singh, and R. Sirohi, "Optimization of freeze-drying process parameters for qualitative evaluation of button mushroom (Agaricus bisporus)using response surface methodology," Journal of Food Quality, vol. 2017, Article ID 5043612, 6 pages, 2017.

[10] Y. Roos and M. Karel, "Amorphous state and delayed ice formation in sucrose solutions," International Journal of Food Science \& Technology, vol. 26, no. 6, pp. 553-566, 1991.

[11] Y. H. Roos, "Frozen state transitions in relation to freeze drying," Journal of Thermal Analysis and Calorimetry, vol. 48, no. 3, pp. 535-544, 1997.

[12] H. T. Chan, S. C. M. Kwok, and C. W. Q. Lee, "Sugar composition and invertase activity in lychee," Journal of Food Science, vol. 40, no. 4, pp. 772-774, 1975.

[13] M. C. Wu and C. S. Chen, "A research note: Effect of sugar types and citric acid content on the quality of canned lychee," Journal of Food Quality, vol. 22, no. 4, pp. 461-469, 1999.

[14] V. A. Drebushchak, A. G. Ogienko, and A. S. Yunoshev, "Metastable eutectic melting in the NaCl-H2O system," Thermochimica Acta, vol. 647, pp. 94-100, 2017.

[15] D. Witrowa-Rajchert and M. Rzaca, "Effect of drying method on the microstructure and physical properties of dried apples," Drying Technology, vol. 27, no. 7, pp. 903-909, 2009.

[16] L.-L. Huang, F. Qiao, and C.-F. Fang, "Studies on the Microstructure and Quality of Iron Yam Slices during Combined Freeze Drying and Microwave Vacuum Drying," Journal 
of Food Processing and Preservation, vol. 39, no. 6, pp. 2152-2160, 2015.

[17] H. C. P. Karunasena, P. Hesami, W. Senadeera, Y. T. Gu, R. J. Brown, and A. Oloyede, "Scanning Electron Microscopic Study of Microstructure of Gala Apples During Hot Air Drying," Drying Technology, vol. 32, no. 4, pp. 455-468, 2014.

[18] C. Niamnuy, S. Devahastin, and S. Soponronnarit, "Some recent advances in microstructural modification and monitoring of foods during drying: A review," Journal of Food Engineering, vol. 123, pp. 148-156, 2014.

[19] B.-G. Xu, M. Zhang, B. Bhandari, X.-F. Cheng, and M. N. Islam, "Effect of ultrasound-assisted freezing on the physico-chemical properties and volatile compounds of red radish," Ultrasonics Sonochemistry, vol. 27, no. 1, pp. 316-324, 2015.

[20] M. N. Islam, M. Zhang, H. Liu, and C. Xinfeng, "Effects of ultrasound on glass transition temperature of freeze-dried pear (Pyrus pyrifolia) using DMA thermal analysis," Food and Bioproducts Processing, vol. 94, pp. 229-238, 2015.

[21] A. Fijalkowska, M. Nowacka, A. Wiktor, M. Sledz, and D. Witrowa-Rajchert, "Ultrasound as a Pretreatment Method to Improve Drying Kinetics and Sensory Properties of Dried Apple," Journal of Food Process Engineering, vol. 39, no. 3, pp. 256-265, 2016.

[22] L. L. Huang, F. Qiao, and D. M. Fan, "Microstructure changing and moisture removing of lychee during microwave vacuum drying," International Journal of Agricultural and Biological Engineering, vol. 9, no. 3, pp. 162-169, 2016.

[23] R. M. Syamaladevi, S. S. Sablani, J. Tang, J. Powers, and B. G. Swanson, "State diagram and water adsorption isotherm of raspberry (Rubus idaeus)," Journal of Food Engineering, vol. 91, no. 3, pp. 460-467, 2009.

[24] L.-L. Huang, M. Zhang, A. S. Mujumdar, and R.-X. Lim, "Comparison of four drying methods for re-structured mixed potato with apple chips," Journal of Food Engineering, vol. 103, no. 3, pp. 279-284, 2011.

[25] A. I. Liapis and R. Bruttini, "Freeze drying," in Handbook of Industrial Drying, A. S. Mujumdar, Ed., CRC Press, Boca Raton, FL, USA, 4th edition, article 259, 2006.

[26] B. R. Bhandari and T. Howes, "Implication of glass transition for the drying and stability of dried foods," Journal of Food Engineering, vol. 40, no. 1, pp. 71-79, 1999.

[27] X. Duan, X. Yang, G. Ren, Y. Pang, L. Liu, and Y. Liu, “Technical aspects in freeze-drying of foods," Drying Technology, vol. 34, no. 11, pp. 1271-1285, 2016.

[28] C. W. Huang, X. H. Liang, and X. W. Fan, "Design of instrument for measuring eutectic and co-melting point of freeze-drying material," Chinese Medical Equipment Journal, vol. 31, no. 4, pp. 15-17, 2010 (Chinese).

[29] R. Wang, M. Zhang, and A. S. Mujumdar, "Effects of vacuum and microwave freeze drying on microstructure and quality of potato slices," Journal of Food Engineering, vol. 101, no. 2, pp. 131-139, 2010.

[30] X. Duan, M. Zhang, X. Li, and A. Mujumdar, "Ultrasonically enhanced osmotic pretreatment of sea cucumber prior to microwave freeze drying," Drying Technology, vol. 26, no. 4, pp. 420-426, 2008.

[31] Y. Wang, M. Zhang, B. Adhikari, A. S. Mujumdar, and B. Zhou, "The Application of Ultrasound Pretreatment and PulseSpouted Bed Microwave Freeze Drying to Produce Desalted Duck Egg White Powders," Drying Technology, vol. 31, no. 15, pp. 1826-1836, 2013. 

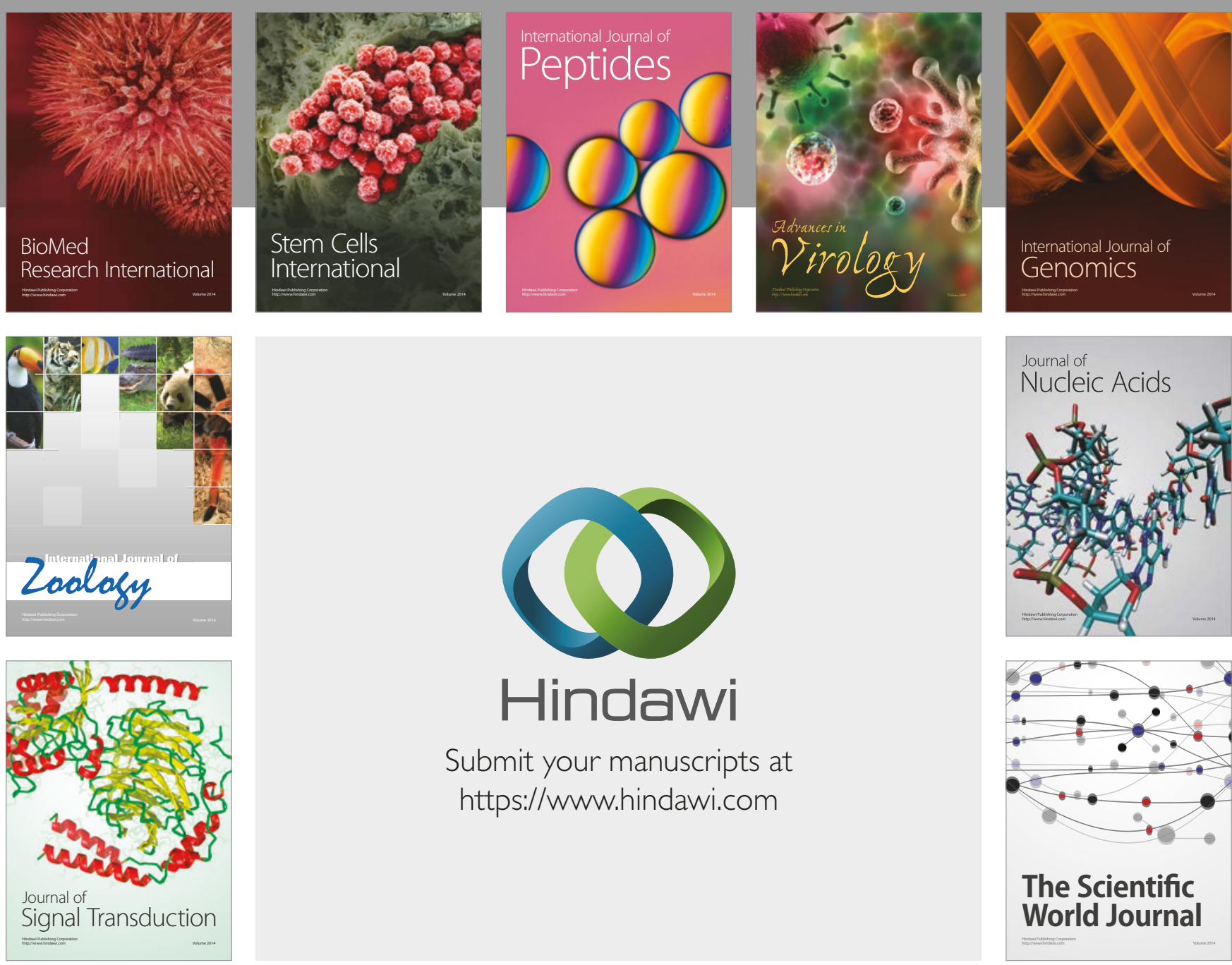

Submit your manuscripts at

https://www.hindawi.com
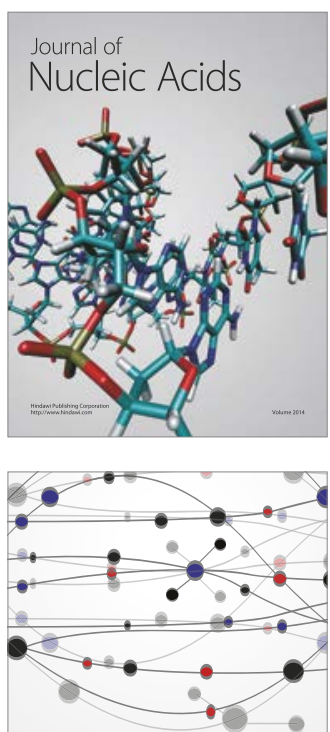

The Scientific World Journal

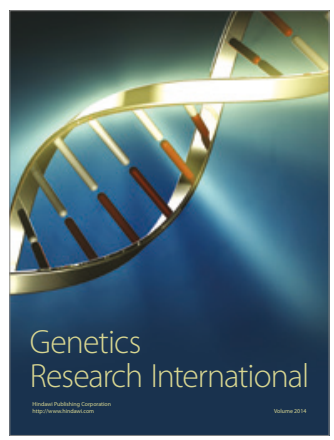

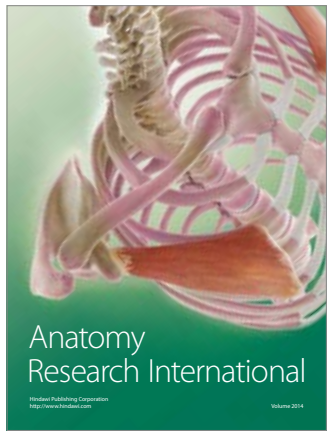

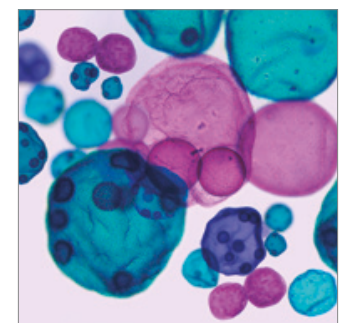

International Journal of Microbiology
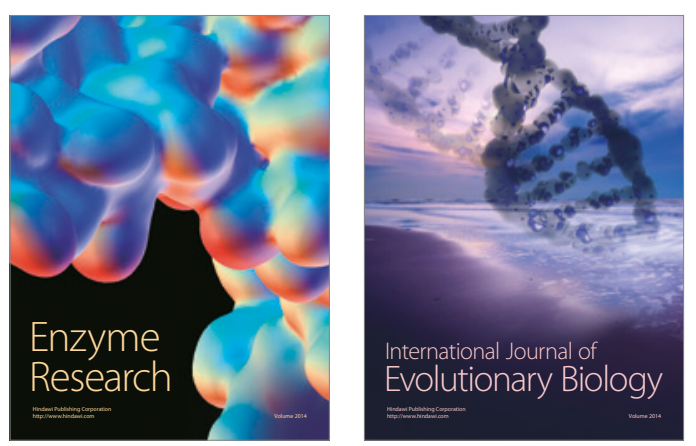
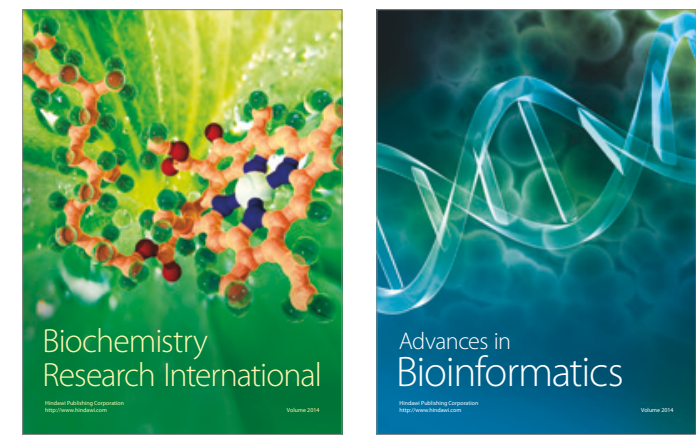

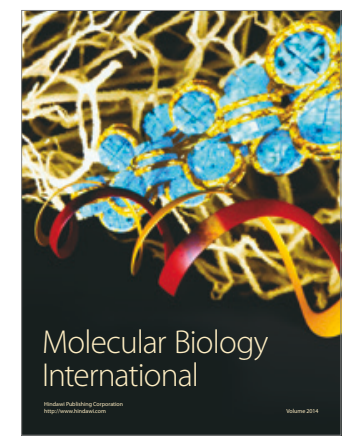

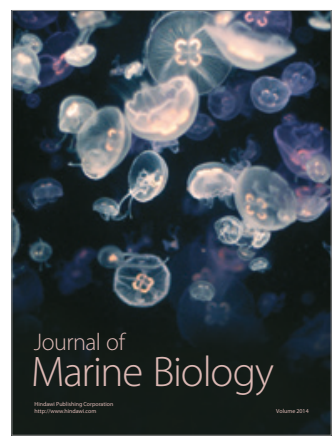

